\title{
Utilização das técnicas cirúrgicas em periodontia: Revisão da literatura
}

\author{
Use of surgical techniques in periodontics: Literature review
}

Uso de técnicas quirúrgicas en periodoncia: revisión de la literature

Karola Mayra dos Santos Vicente ORCID: https://orcid.org/0000-0001-5518-3893 Centro Universitário Maurício de Nassau, Brasil E-mail: karolavicente@hotmail.com

Beatriz Reis de Oliveira

ORCID: https://orcid.org/0000-0003-4040-5195 Universidade Tiradentes, Brasil

E-mail: beatrizreisoliveiraa@hotmail.com

Elias Abraão Dos Reis Silva

ORCID: https://orcid.org/0000-0003-0549-6706

Universidade Tiradentes, Brasil

E-mail: e.abraao.rs@gmail.com

Gabriel Santana Santos

ORCID: https://orcid.org/0000-0002-6539-7120

Universidade Tiradentes, Brasil

E-mail: gabrielsnt9@hotmail.com

Gustavo Dultra Todt

ORCID: https://orcid.org/0000-0003-2208-8317

Universidade Tiradentes, Brasil

E-mail: gugatodt@hotmail.com

Ísis Suzanne Crisóstomo dos Santos

ORCID: https://orcid.org/0000-0002-5991-0143

Universidade Tiradentes, Brasil

E-mail: isissuzanne_@hotmail.com

Joana Machado Costa

ORCID: https://orcid.org/0000-0002-4421-2713

Universidade Tiradentes, Brasil

E-mail: jmcbpinheiro@gmail.com

José Augusto de Oliveira Neto

ORCID: https://orcid.org/0000-0001-9354-0900

Universidade Tiradentes, Brasil

E-mail: joseaugusto19901@gmail.com

Liana Gois Seabra

ORCID: https://orcid.org/0000-0001-7089-3658

Universidade Tiradentes, Brasil

E-mail: liana_gois@hotmail.com

Louise Cristina Santos

ORCID: https://orcid.org/0000-0001-7458-975X Universidade Tiradentes, Brasil

E-mail: louise_cristina123@hotmail.com

Luciana Lima de Gois

ORCID: https://orcid.org/0000-0002-2912-9807 Universidade Tiradentes, Brasil

E-mail: luciana.lima00@souunit.com.br

Mariana Alves Rocha Souza

ORCID: https://orcid.org/0000-0002-4176-8206 Universidade Tiradentes, Brasil

E-mail: mariana_aars@hotmail.com

Mário Gomes Brandão Neto

ORCID: https://orcid.org/0000-0002-8353-3085 Universidade Tiradentes, Brasil

E-mail: mariovent2014@ hotmail.com

Nathália Ferreira Santos

ORCID: https://orcid.org/0000-0003-1770-2108

Universidade Tiradentes, Brasil

E-mail: nath_ferreira06@hotmail.com

Noely Mayara Bispo de Souza

ORCID: https://orcid.org/0000-0002-1992-0179

Universidade Tiradentes, Brasi

E-mail: mayara.bispo@ hotmail.com 
Ódila Vanessa Amaral de Almeida

ORCID: https://orcid.org/0000-0002-4728-8502

Universidade Tiradentes, Brasil

E-mail: odila601@ outlook.com

Tiago de Oliveira Goulart

ORCID: https://orcid.org/0000-0003-0157-6004 Universidade Tiradentes, Brasil

E-mail: tiago.ogoulart@outlook.com

Thalwylla Reiler Morato dos Reis Moreira

ORCID: https://orcid.org/0000-0001-5632-2322 Universidade Federal do Alagoas, Brasil E-mail: thalwyllamorato@yahoo.com.br

Valéria Vilhena Butarelli Nobre

ORCID: https://orcid.org/0000-0002-0187-096X Universidade Tiradentes, Brasil E-mail: vbutarelli09@gmail.com

Vitória Virgínia Maria Machado Vanderley ORCID: https://orcid.org/0000-0001-8972-3595 Universidade Tiradentes, Brasil

E-mail: vitoriavirginiamachado@gmail.com

Rafaella Bastos Leite

ORCID: https://orcid.org/0000-0002-3304-120X Centro Universitário Maurício de Nassau, Brasil E-mail: rafaella_bastos@hotmail.com

\title{
Resumo
}

A cirurgia periodontal é uma parte importante do tratamento periodontal e deve ser levada em consideração quando a terapia convencional não elimina o problema periodontal. Também apresenta-se indicada em alguns casos estéticos ou casos em que seja necessário melhorar o acesso à determinada área para dessa forma realizar o correto plano de tratamento. $\mathrm{O}$ objetivo do presente estudo é apresentar as principais indicações das técnicas cirúrgicas periodontais e detalhar os procedimentos apresentados na literatura. Trata-se de uma revisão de literatura, utilizando como base livros e artigos científicos presentes no PubMed, Scielo e Google Acadêmico. É importante salientar que a terapia periodontal não cirúrgica preceda o tratamento periodontal cirúrgico. A associação entre a cirurgia mucogengival e enxertos, demonstrou resultado positivo no tratamento de recessões gengivais, assim como a cirurgia regenerativa com uso de biomateriais apresentou eficácia no tratamento de defeitos ósseos periodontais. A cirurgia periodontal pode ser empregada como uma importante ferramenta no tratamento de diversos problemas periodontais.

Palavras-chave: Doenças Periodontais; Retração Gengival; Retalhos Cirúrgicos.

\begin{abstract}
Periodontal surgery is an important part of periodontal treatment and must be taken into account when conventional therapy does not eliminate the periodontal problem. It is also indicated in some aesthetic cases or cases in which it is necessary to improve access to a certain area in order to carry out the correct treatment plan. The aim of the present study is to present the main indications for periodontal surgical techniques and to detail the procedures presented in the literature. This is a literature review, based on books and scientific articles present in PubMed, Scielo and Google Scholar. It is important to note that non-surgical periodontal therapy precedes surgical periodontal treatment. The association between mucogingival surgery and grafts has shown a positive result in the treatment of gingival recessions, as well as regenerative surgery with the use of biomaterials has shown efficacy in the treatment of periodontal bone defects. Periodontal surgery can be used as an important tool in the treatment of various periodontal problems.
\end{abstract}

Keywords: Periodontal Diseases; Gingival Recession; Surgical Flaps.

\section{Resumen}

La cirugía periodontal es una parte importante del tratamiento periodontal y debe tenerse en cuenta cuando la terapia convencional no elimina el problema periodontal. También está indicado en algunos casos estéticos o casos en los que sea necesario mejorar el acceso a una determinada zona para poder llevar a cabo el correcto plan de tratamiento. El objetivo del presente estudio es presentar las principales indicaciones de las técnicas quirúrgicas periodontales y detallar los procedimientos presentados en la literatura. Se trata de una revisión bibliográfica, basada en libros y artículos científicos presentes en PubMed, Scielo y Google Scholar. Es importante señalar que la terapia periodontal no quirúrgica precede al tratamiento periodontal quirúrgico. La asociación entre cirugía mucogingival e injertos ha mostrado un resultado positivo en el tratamiento de las recesiones gingivales, así como la cirugía regenerativa con el uso de biomateriales ha mostrado eficacia en el tratamiento de los defectos óseos periodontales. La cirugía periodontal se puede utilizar como una herramienta importante en el tratamiento de diversos problemas periodontales.

Palabras clave: Enfermedades Periodontales; Recesión Gingival; Colgajos Quirúrgicos. 


\section{Introdução}

A periodontite caracteriza-se como uma inflamação crônica causada por micro-organismos relacionados ao biofilme oral, gerando uma destruição gradual dos tecidos de suporte dentário. Apresenta como características clínicas a perda de suporte tecidual, perda de inserção clínica e perda óssea alveolar (Papapanou, 2018). Trata-se de um problema de saúde com alta prevalência, que pode exibir um bom prognóstico se for tratado precocemente, caso isso não aconteça, pode causar a perda dentária e afetar a função mastigatória do indivíduo acometido, prejudicando também a sua estética do sorriso e consequentemente a sua qualidade de vida (Papapanou, 2018; Slots, 2017).

Esta patologia, caracteriza-se pela perda de inserção interproximal em dois ou mais locais não adjacentes ou perda de inserção maior ou igual a 3mm em dois ou mais dentes, sem ter relação com recessões gengivais traumáticas; cárie cervical; perda de inserção na distal do segundo molar ocasionada pela extração ou mal posicionamento do terceiro molar; lesão endodôntica com drenagem pelo periodonto marginal; fratura vertical de raiz (Papapanou, 2018; Slots, 2017). Sendo classificada em estágios I, II, III, IV de Miller de acordo com a severidade relacionada à perda de inserção clínica e graus A, B e C de acordo com evidências ou risco de progressão da doença (Steffens, 2018; Papapanou, 2018; Tonetti, 2019).

O tratamento periodontal deve ser iniciado com uma modalidade não-cirúrgica, que envolve a orientação de higiene oral e raspagem e alisamento coronorradicular, visando dessa forma minimizar a inflamação gengival, controlar o biofilme oral e facilitar a visibilidade clínica. Em muitos casos o tratamento não-cirúrgico pode ser o tratamento definitivo, porém em alguns casos a cirurgia periodontal se torna necessária (Steffens, 2018; Papapanou, 2018; Tonetti, 2019).

$\mathrm{O}$ tratamento da periodontite requer além das modalidades terapêuticas empregadas pelo profissional, o autocuidado por parte do paciente, através do controle do biofilme oral supragengival. As bolsas periodontais, geradas por negligências da higienização oral, apresentam-se como uma das características mais presentes na doença periodontal, estas são definidas como um aprofundamento patológico do sulco gengival (Graziani, 2017).

A diminuição de bolsas periodontais é um dos objetivos principais do tratamento periodontal, esta pode ser conseguida através de modalidades cirúrgicas ou não-cirúrgicas. A não resolução do problema, através de bolsas residuais pode comprometer os resultados esperados. É difícil de estabelecer qual paciente necessita de tratamento cirúrgico ou não cirúrgico. Porém, foi observado que o tratamento cirúrgico tem excelentes resultados em termos de diminuição de bolsas periodontais (Graziany, 2017).

A cirurgia periodontal é composta pelo conjunto de procedimentos cirúrgicos que visam manter a saúde do complexo mucogengival e regenerar os tecidos periodontais. Apresenta vários benefícios, tais como: facilitar o acesso ao biofilme subgengival, melhorar a visibilidade, adequar a morfologia gengival, auxiliar o paciente a manter a higiene adequada, criar acesso adequado à superfície radicular, eliminar bolsas profundas e transformar o fenótipo gengival (Steffens, 2018; Papapanou, 2018; Tonetti, 2019). Dentre as opções cirúrgicas destacam-se os procedimentos mucogengivais e os regenerativos.

As cirurgias mucogengivais atuam corrigindo defeitos na forma, defeitos traumáticos ou de desenvolvimento na gengiva ou mucosa alveolar, agindo positivamente na estética do paciente, minimizando também o nível de sensibilidade dentária nas áreas que estavam antes descobertas (Steffens, 2018; Papapanou, 2018; Tonetti, 2019).

As cirurgias regenerativas promovem a regeneração periodontal, os métodos empregados para regeneração devem preservar os tecidos de suporte periodontal e respeitar a sequência natural dos eventos biológicos que acontecem no periodonto (Siaili, 2018).

As cirurgias regenerativas podem ser divididas em procedimentos associados ou não-associados a enxertos. Dentre os materiais utilizados em cirurgias regenerativas associadas a enxertos, estão o autoenxerto, aloenxerto e o xenoenxerto. Tais materiais podem ser também avaliados em relação ao potencial osteogênico, osteoindutivo e osteocondutor (Steffens, 2018; 
Papapanou, 2018; Tonetti, 2019).

Portanto, esse estudo tem como objetivo avaliar as principais técnicas cirúrgicas mucogengivais e regenerativas em periodontia, observando as suas principais indicações na prática clínica odontológica, visando contribuir para uma melhora no planejamento, diagnóstico e elaboração de um correto plano de tratamento para os problemas periodontais que necessitam de intervenção cirúrgica, alcançando-se resultados mais positivos.

\section{Metodologia}

Este estudo trata-se de uma revisão de literatura, de caráter qualitativo, utilizando como base livros, revistas científicas e artigos científicos presentes na base de dados PubMed, Lilacs, Scielo e Google Acadêmico. Foi realizada a análise de artigos relacionados à temática da pesquisa, buscando-se assim fortalecer a construção do referencial teórico. Utilizaram-se os descritores "Guided Tissue Regeneration", "Periodontal diseases”, "Gingival Recession”, "Plastic Surgery”, "Surgical Flaps", "Periodontal Pocket", assim como o operador booleano and. Foram selecionados textos em português ou em inglês.

Os critérios de inclusão utilizados foram artigos e livros publicados em português ou em inglês, que se enquadraram ao tema da pesquisa e que apresentaram metodologia empregada de forma detalhada. Foram excluídos trabalhos em duplicata ou que não se enquadrassem à metodologia empregada. Além disso, foi realizada a leitura detalhada e seleção do material de escolha, com a finalidade de fundamentar um debate acerca das principais técnicas cirúrgicas mucogengivais e regenerativas utilizadas em periodontia.

\section{Resultados}

Os descritores foram utilizados separadamente e alguns em associação para realizar a busca dos artigos, foram selecionados inicialmente 60 artigos, dos quais 12 foram utilizados para elaboração do presente trabalho. Dos 12 artigos, 10 tratam de cirurgia periodontal mucogengival e 2 tratam de cirurgia periodontal regenerativa em associação à cirurgia mucogengival. No quadro 1 estão descritos artigos sobre cirurgia periodontal, em número de 12, organizados de acordo com o autor/ano, tipo de estudo, número de pacientes, tipo de procedimento e principais resultados.

Quadro 1 - Artigos selecionados sobre cirurgias periodontais, de acordo com autor/ano, tipo de estudo, número de pacientes, tipo de procedimento e principais resultados, de 2015-2020.

\begin{tabular}{|c|c|c|c|c|}
\hline Autor/ Ano & Tipo de Estudo & $\begin{array}{l}\text { Número de } \\
\text { Pacientes }\end{array}$ & Tipo de Procedimento & Principais resultados \\
\hline Zucchelli (2016) & $\begin{array}{l}\text { Ensaio clínico duplo- } \\
\text { cego, randomizado e } \\
\text { controlado. }\end{array}$ & 50 pacientes. & $\begin{array}{l}\text { Retalho avançado } \\
\text { coronalmente } \\
\text { triangular } \\
\text { trapezoidal. }\end{array}$ & $\begin{array}{l}\text { Nenhuma } \\
\text { significativa foi observada } \\
\text { no nível de cobertura } \\
\text { radicular entre os dois } \\
\text { grupos. Após a fase inicial } \\
\text { de higiene oral e nos exames } \\
\text { pós-tratamento, todos os } \\
\text { indivíduos apresentaram } \\
\text { baixa frequência de placa e } \\
\text { sangramento gengival. A } \\
\text { cura transcorreu sem } \\
\text { intercorrências em todos os } \\
\text { casos tratados com retalho } \\
\text { coronal avançado. }\end{array}$ \\
\hline
\end{tabular}




\begin{tabular}{|c|c|c|c|c|c|}
\hline Rath (2016) & $\begin{array}{l}\text { Relato de } \\
\text { Clínico. }\end{array}$ & Caso & 1 paciente. & $\begin{array}{l}\text { Cirurgia mucogengival } \\
\text { em dois estágios com } \\
\text { autotransplante } \\
\text { gengival livre, uso de } \\
\text { membrana de colágeno } \\
\text { e retalho avançado } \\
\text { coronalmente. }\end{array}$ & $\begin{array}{l}\text { No primeiro estágio } \\
\text { cirúrgico houve uma } \\
\text { cobertura de } 5 \mathrm{~mm} \text { de raiz e } \\
\text { aumento de } 4 \mathrm{~mm} \text { na gengiva } \\
\text { inserida. No segundo estágio } \\
\text { cirúrgico houve um aumento } \\
\text { adicional de } 7 \mathrm{~mm} \text { de } \\
\text { cobertura radicular e } 4 \mathrm{~mm} \\
\text { de gengiva inserida após seis } \\
\text { meses. }\end{array}$ \\
\hline $\begin{array}{l}\text { Garces-Mcintyre } \\
\text { (2017) }\end{array}$ & $\begin{array}{l}\text { Estudo } \\
\text { Prospectivo. }\end{array}$ & Clínico & 20 pacientes. & $\begin{array}{lr}\text { Retalho } & \text { avançado } \\
\text { coronalmente } & \text { em } \\
\text { associação } & \text { com } \\
\text { enxerto de } & \text { tecido } \\
\text { conjuntivo. } & \end{array}$ & $\begin{array}{l}\text { Uma diminuição } \\
\text { estatisticamente significativa } \\
\text { na profundidade das } \\
\text { recessões foi observada no } \\
\text { final do período de } \\
\text { avaliação. A porcentagem } \\
\text { média de cobertura da raiz } \\
\text { foi } 93,44 \% \text { para todas as } \\
\text { recessões tratadas, e a } \\
\text { cobertura completa da raiz } \\
\text { foi alcançada em } 65 \% \text { de } \\
\text { casos (13 pacientes). }\end{array}$ \\
\hline Goyal (2019) & $\begin{array}{l}\text { Ensaio } \\
\text { Randomizado. }\end{array}$ & Clinico & 10 pacientes. & $\begin{array}{l}\text { Enxerto gengival livre } \\
\text { em etapa única. }\end{array}$ & $\begin{array}{l}\text { Sucesso do enxerto gengival } \\
\text { livre. Ao final de } 9 \text { meses } \\
\text { cobertura de } 82 \% \text {, ganho de } \\
\text { inserção clínica, ganho de } \\
\text { largura da gengiva inserida e } \\
\text { melhora na profundidade de } \\
\text { sondagem. }\end{array}$ \\
\hline Dias-Ribeiro (2020) & $\begin{array}{l}\text { Relato de } \\
\text { Clínico. }\end{array}$ & Caso & 1 paciente. & Enxerto gengival livre. & 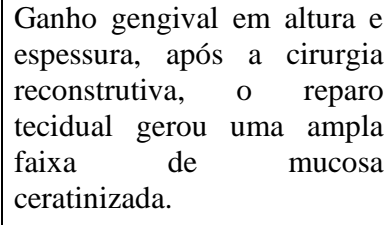 \\
\hline Cairo. (2016) & $\begin{array}{l}\text { Ensaio } \\
\text { Randomizado } \\
\text { Controlado. }\end{array}$ & Clínico & 32 pacientes. & $\begin{array}{l}\text { Retalho avançado } \\
\text { coronalmente com ou } \\
\text { sem enxerto de tecido } \\
\text { conjuntivo. }\end{array}$ & $\begin{array}{l}\text { Após } 12 \text { meses, } 69 \% \text { dos } \\
\text { pacientes tratados com } \\
\text { retalho avançado } \\
\text { coronalmente }+ \text { enxerto de } \\
\text { tecido conjuntivo tiveram } \\
\text { cobertura completa de todas } \\
\text { as recessões tratadas, no } \\
\text { grupo de retalho avançado } \\
\text { coronalmente apenas } 25 \% \\
\text { dos pacientes obtiveram } \\
\text { cobertura total de todas as } \\
\text { recessões. }\end{array}$ \\
\hline Agudio (2019) & $\begin{array}{l}\text { Série de } \\
\text { Retrospectivos }\end{array}$ & Casos & 52 pacientes & Retalho gengival livre & $\begin{array}{llr}\text { A faixa de } & \text { tecido } \\
\text { queratinizado } & \text { pode } \\
\text { influenciar } & & \text { o } \\
\text { desenvolvimento de lesões } \\
\text { cervicais não cariosas. } \\
\text { Locais com rais tecido } \\
\text { queratinizado aderido }<2 \text { mm } \\
\text { ou que apresentaram um } \\
\text { biótipo periodontal fino } \\
\text { tiveram aproximadamente } \\
3,5 \text { vezes a chance de } \\
\text { desenvolver lesões cervicais } \\
\text { não cariosas. }\end{array}$ \\
\hline
\end{tabular}




\begin{tabular}{|c|c|c|c|c|c|}
\hline Santoro (2016) & $\begin{array}{l}\text { Relato de } \\
\text { Clínicos }\end{array}$ & Casos & 2 pacientes & $\begin{array}{l}\text { Cirurgia regenerativa e } \\
\text { mucogengival }\end{array}$ & $\begin{array}{l}\text { Em ambos os casos foi } \\
\text { observada melhora na } \\
\text { cobertura radicular, nível de } \\
\text { inserção clínica de } 5 \mathrm{~mm} \text { e } \\
\text { profundidade de sondagem } \\
\text { de } 3 \mathrm{~mm} \text {. }\end{array}$ \\
\hline Cortellini (2016) & $\begin{array}{l}\text { Ensaio } \\
\text { Randomizado }\end{array}$ & Clínico & 45 pacientes & $\begin{array}{l}\text { Cirurgia regenerativa e } \\
\text { cirurgia a retalho }\end{array}$ & $\begin{array}{l}\text { Todos os dentes regenerados } \\
\text { se mantiveram em função } 20 \\
\text { anos após tratamento inicial, } \\
\text { enquanto } 85,7 \% \text { dos dentes } \\
\text { tratados com cirurgia a } \\
\text { retalho sobreviveram ao } \\
\text { longo do tempo. }\end{array}$ \\
\hline Reino (2015) & $\begin{array}{l}\text { Estudo Com } \\
\text { Randomizado }\end{array}$ & parativo & 20 pacientes & $\begin{array}{l}\text { Retalho avançado } \\
\text { coronalmente }+ \text { matriz } \\
\text { de colágeno suíno e } \\
\text { retalho estendido + } \\
\text { matriz de colágeno } \\
\text { suíno }\end{array}$ & $\begin{array}{l}\text { A cicatrização da ferida } \\
\text { ocorreu sem intercorrências } \\
\text { em todos os casos, sem } \\
\text { exposição do enxerto, houve } \\
\text { ganho de inserção clínica, } \\
\text { diminuição na recessão } \\
\text { gengival com uma diferença } \\
\text { estatisticamente } \\
\text { significativa. }\end{array}$ \\
\hline Ahmedbeyli (2018) & $\begin{array}{l}\text { Ensaio } \\
\text { randomizado }\end{array}$ & clínico & 22 pacientes & $\begin{array}{l}\text { Retalho posicionado } \\
\text { lateralmente+ enxerto } \\
\text { de matriz dérmica } \\
\text { acelular }\end{array}$ & $\begin{array}{l}\text { De } 11 \text { defeitos em cada } \\
\text { grupo, a completa cobertura } \\
\text { da raiz foi observada em } 8 \\
\text { pacientes no grupo retalho } \\
\text { posicionado lateralmente + } \\
\text { enxerto de matriz dérmica } \\
\text { acelular }(72,73 \%) \text { e } 5 \\
\text { pacientes }(45,45 \%) \text { no grupo } \\
\text { de retalho posicionado } \\
\text { lateralmente }(p<0,05) \text {. }\end{array}$ \\
\hline Bansal (2016) & $\begin{array}{l}\text { Ensaio } \\
\text { Randomizado } \\
\text { Controlado }\end{array}$ & Clínico & 10 pacientes & $\begin{array}{l}\text { Retalho avançado } \\
\text { coronalmente com e } \\
\text { sem massa de vidro } \\
\text { bioativo }\end{array}$ & $\begin{array}{l}\text { Seis meses após a cirurgia, a } \\
\text { recessão gengival mostrou } \\
\text { redução significativa tanto } \\
\text { no grupo de teste quanto no } \\
\text { grupo controle }(2,0 \pm 0,47 \\
\text { mm e } 2,3 \pm 0,48 \text {, } \pm \text { sem } \\
\text { respectivamente p<0,05) sem } \\
\text { diferença entre os grupos. As } \\
\text { raízes expostas foram } \\
\text { cobertas } 72 \% \text { (teste) e } 79 \% \\
\text { (controle). O ganho no nível } \\
\text { de inserção clínica também } \\
\text { foi significativo (teste: } \\
2,7 \pm 0,67 m m ; \text { controle: } 2,8 \pm \\
0,78 \mathrm{~mm} ; \mathrm{p}<0,05) \text {. }\end{array}$ \\
\hline
\end{tabular}

Fonte: Autores (2020).

\section{Discussão}

Zucchelli (2016) trazem como principais indicações para cirurgia de recobrimento radicular as razões estéticas, hipersensibilidade dental, abrasão ou cáries de raiz, aumento de tecido queratinizado, inconsistência ou desarmonia da margem gengival que podem fazer com que o paciente não realize uma técnica adequada de escovação. Graziani (2017) em sua pesquisa, mostraram que o tratamento periodontal cirúrgico se apresenta indicado em recessões maiores ou iguais a $5 \mathrm{~mm}$ ou em locais com profundidade de sondagem maior ou igual a $6 \mathrm{~mm}$.

Rath (2016) em seus trabalhos envolvendo cirurgia mucogengival em dois estágios com enxerto gengival livre no tratamento de recessões Classe III de Miller, obtiveram uma cobertura de $7 \mathrm{~mm}$ nas raízes e aumento de $4 \mathrm{~mm}$ na gengiva 
inserida dos pacientes tratados seis meses após o tratamento. Enquanto Garces-Mcintyre (2017) em seus estudos prospectivos de retalhos coronais avançados combinados com enxerto de tecido conjuntivo para cobertura de recessões gengivais, tiveram como resultado uma cobertura média de $93,44 \%$ em todas as recessões tratadas e $65 \%$ dos casos tiveram uma cobertura completa. Sendo assim, nos dois estudos a associação entre procedimentos cirúrgicos mostrou-se positiva, melhorando os resultados esperados a longo prazo.

O enxerto gengival livre é um procedimento eficaz na cobertura radicular e também tem um importante papel como método auxiliar na prevenção de lesões cervicais não cariosas, assim como apresentam em seus estudos Goyal (2019) que citam em seu ensaio clínico randomizado o enxerto gengival livre como um procedimento útil na cobertura radicular de pacientes que apresentam recessões gengivais classe I e II de Miller, onde obtive-se uma cobertura radicular de 82\%, além disso, também houve diminuição da profundidade de sondagem e ganho no nível de inserção clínica nos pacientes tratados e Agudio (2019), que apresentam o enxerto gengival livre como uma opção para reduzir os riscos de ocorrência de lesões cervicais não cariosas, tendo como resultado que a gengiva queratinizada pode influenciar sua ocorrência e que locais que apresentaram menos de $2 \mathrm{~mm}$ de gengiva queratinizada anexa ou tiveram um fenótipo periodontal fino, obtiveram uma maior chance de desenvolver lesões cervicais não cariosas, aproximadamente 3,5 vezes chances de apresentar lesões cervicais não cariosas $>0,5 \mathrm{~mm}$.

Dias-Ribeiro (2020) apresentam o emprego do enxerto gengival livre anteriormente a cirurgias reconstrutivas para corrigir defeitos nos tecidos moles gengivais, em seu relato de caso, o enxerto gengival misto (enxerto gengival livre com pedículo de tecido conjuntivo) foi empregado para correção de um defeito peri-implantar em região anterior de maxila, onde obteve-se uma ótima efetividade, ganho de gengiva tanto em altura como em espessura, proporcionando dessa forma um bom aspecto gengival. Sendo assim, a utilização de enxerto gengival livre apresentou-se satisfatória na correção do defeito periimplantar, precedendo a cirurgia reconstrutiva.

Reino (2015), em seu estudo comparativo randomizado com duas técnicas cirúrgicas para otimizar a cobertura radicular, no qual 20 pacientes com recessões classe I de Miller foram submetidos à cirurgia com retalho avançado coronalmente + matriz de colágeno suíno ou retalho estendido + matriz de colágeno suíno, tendo como resultado boa cicatrização da ferida, sem exposição do enxerto, ganho de inserção clínica e boa diminuição das recessões gengivais. Os mesmos autores também concluíram que o retalho estendido obteve resultados superiores aos do retalho avançado coronalmente no que se refere ao tratamento das recessões.

Cairo (2016) em sua pesquisa sobre a eficácia do retalho avançado coronalmente em associação ou não com enxerto de tecido conjuntivo no tratamento de múltiplas recessões gengivais adjacentes, observaram que em $69 \%$ dos pacientes com recessões gengivais que foram tratados com retalho avançado coronalmente e com enxerto de tecido conjuntivo em associação, apresentaram uma cobertura total das recessões, enquanto em pacientes apenas tratados com retalho avançado coronalmente somente $25 \%$ dos pacientes tiveram uma cobertura total, apresentando assim, uma maior porcentagem de sucesso quando os procedimentos foram utilizados em associação.

Bansal (2016) em seu estudo clínico randomizado controlado com retalhos avançados coronalmente com ou sem massa de vidro bioativo, obtiveram uma boa redução no grau das recessões gengivais no grupo de teste e controle $(\mathrm{p}<0,05)$, recobrimento radicular de $72 \% \pm 15,81 \%$ no grupo de teste e $79 \% \pm 18,94 \%$ no grupo controle e ganho no nível de inserção clínica em ambos os grupos (com e sem enxerto). Assim, o vidro bioativo mostrou-se como uma opção de material aloplástico disponível para enxertos, com boa efetividade quando utilizado em associação com o retalho avançado coronalmente.

Santoro (2016) em seu estudo, combinando o tratamento regenerativo e mucogengival em defeitos intraósseos associados à recessão gengival, obtiveram resultados que mostram que tais procedimentos são previsíveis, onde há ganho de inserção clínica e aumento do nível ósseo, gerando também uma boa cobertura radicular. Ao mesmo tempo que Cortellini 
(2016) em seu ensaio clínico randomizado com procedimentos periodontais regenerativos e procedimentos a retalho em defeitos intraósseos, tiveram como resultado uma boa evolução dos defeitos tratados principalmente com a regeneração periodontal, no qual todos os dentes permaneceram em função, enquanto em defeitos tratados apenas com retalho, $(85,7 \%)$ dos dentes permaneceram em função 20 anos após o tratamento inicial. Dessa maneira, os autores destacam a superioridade dos procedimentos regenerativos no tratamento de defeitos intraósseos, onde houve ganho no nível de inserção clínica, ausência de perda dentária e menos intervenções ao longo do tempo.

Ahmedbeyli (2018) em seu ensaio clínico randomizado sobre retalho posicionado lateralmente com enxerto de matriz dérmica acelular no tratamento de recessões gengivais maxilares, envolvendo 22 pacientes, apresentaram como resultados cobertura radicular completa em 8 pacientes no grupo retalho posicionado lateralmente em associação com o enxerto de matriz dérmica acelular $(72,73 \%)$ e 5 pacientes $(45,45 \%)$ no grupo de retalho posicionado lateralmente. Sendo assim, no estudo em questão, o retalho posicionado lateralmente mostrou-se eficaz no tratamento de recessões gengivais maxilares, principalmente quando em associação com o enxerto de matriz dérmica acelular e a utilização de biomateriais também apresentou resultados positivos.

\section{Conclusão}

De acordo com a literatura consultada, foi possível observar que o enxerto gengival livre apresentou resultados positivos no tratamento das recessões gengivais classe I e II de Miller e em cirurgias para correção de defeitos gengivais periimplantares relatadas. Já os retalhos coronais avançados combinados com enxerto de tecido conjuntivo mostraram-se como uma boa opção de tratamento para recessões gengivais. Nesse contexto, vale salientar que a cirurgia regenerativa utilizando biomateriais apresenta-se eficaz no tratamento dos defeitos ósseos periodontais relatados.

\section{Referências}

Agudio, G. (2019). Effect of Gingival Augmentation Procedure (Free Gingival Graft) on Reducing the Risk of Non-Carious Cervical Lesions: a 25-to 30-year Follow-up Study. Journal of Periodontology, 90(11), 1235-1243.

Ahmedbeyli, C. (2018). Laterally positioned flap along with acellular dermal matrix graft in the management of maxillary localized recessions. Clinical Oral Investigations, 23(2), 595-601.

Bansal, A. (2016). Comparative evaluation of coronally advanced flap with and without bioactive glass putty in the management of gingival recession defects: a randomized controlled clinical trial. Journal of the International Academy of Periodontology, 18(1), 7-15.

Cairo, F. (2016). Clinical Efficacy of Coronally Advanced Flap with or without Connective Tissue Graft for the treatment of Multiple Adjacent Gingival Recessions in the Aesthetic Area. A Randomized Controlled Clinical Trial. Journal of Clinical Periodontology, 43(10), 849-856.

Cortellini, P. (2016). Periodontal Regeneration Compared with Access Flap Surgery in Human Intrabony Defects 20-year Follow-up of a Randomized Clinical Trial: Tooth Retention, Periodontitis Recurrence and Costs. Journal of Clinical Periodontology, 44(1), 58-66.

Dias-Ribeiro, A. (2020). Enxerto gengival livre para correção de defeitos de tecido mole previamente a cirurgias reconstrutivas. Archives of Health Investigation, 9(2), 136-140.

Garces-Mcintyre, T. (2017). Coronal advanced flap in combination with a connective tissue graft. Is the thickness of the flap a predictor for root coverage? A prospective clinical study. Journal of Clinical Periodontology, 44(9), 933-940.

Graziani, F. (2017). Nonsurgical and surgical treatment of periodontitis: how many options for one disease? Periodontology 2000, 75(1), 152-188.

Goyal, L. (2019). Free Gingival Graft as a Single Step Procedure for Treatment of Mandibular Miller Class I and II Recession Defects. World journal of plastic surgery, 8(1), 12-17.

Papapanou, P. N. (2018). Periodontitis: Consensus report of workgroup 2 of the 2017 World Workshop on the Classification of Periodontal and Peri-Implant and Diseases and Conditions. Journal of Periodontology, 89(1), 173-182.

Rath, A. (2016). Two-Stage Mucogingival Surgery with Free Gingival Autograft and Biomend Membrane and Coronally Advanced Flap in Treatment of Class III Millers Recession. Case Reports in Dentistry, 2016(1), 1-5.

Reino, D. M. (2015). A Randomized Comparative Study of Two Techniques to Optimize the Root Coverage Using a Porcine Collagen Matrix. Brazilian Dental Journal, 26(5), 445-450. 
Research, Society and Development, v. 10, n. 3, e51410313668, 2021

(CC BY 4.0) | ISSN 2525-3409 | DOI: http://dx.doi.org/10.33448/rsd-v10i3.13668

Santoro, G. (2016). Combined Regenerative and Mucogingival Treatment of Deep Intrabony Defects Associated with Buccal Gingival Recession: Two Case Reports. The International Journal of Periodontics and Restorative Dentistry, 36(6), 849-857.

Siaili, M. (2018). An overview of periodontal regenerative procedures for the general dental practitioner. The Saudi Dental Journal, 30(1), $26-37$.

Slots, J. (2017). Periodontitis: facts, fallacies and the future. Periodontology 2000, 75(1), 7-23.

Steffens, J. P. (2018). Classificação das Doenças e Condições Periodontais e Peri implantares 2018: guia Prático e Pontos-Chave. Revista de Odontologia da UNESP, 47(4), 189-197.

Tonetti, M. S. (2019). Implementation of the New Classification of Periodontal Diseases: Decision Making Algorithms for Clinical Practice and Education. Journal of Clinical Periodontology, 46(4), 398-405.

ZucchellI, G. (2016). Coronally Advanced Flap with Different Designs in the Treatment of Gingival Recession: A Comparative Controlled Randomized Clinical Trial. The International journal of periodontics \& restorative dentistry, 36(3), 319-327. 